\title{
Numerical and experimental analysis of vibratory signals for rolling bearing fault diagnosis
}

\author{
T. Bensana*, S. Mekhilef** \\ *Industrial Mechanics Laboratory (LMI), Badji Mokhtar University, BP 12, Annaba, Algeria \\ E-mail: tbensana@gmail.com \\ **Industrial Mechanics Laboratory (LMI), Badji Mokhtar University, BP 12, Annaba, Algeria \\ cross $^{\text {ref }}$ http://dx.doi.org/10.5755/j01.mech.22.3.11962
}

\section{Nomenclature}

STFT - short-time Fourier transform; WVD - Wigner- Viller distribution; WT- Wavelet transform; PF - product functions; SVD - singular value decomposition; LMD - local mean decomposition; EMD - Empirical mode decomposition; FM - frequency modulated; AM - amplitude modulated; $m_{i}$ - local mean value; $s_{1 n}(t)$ - frequency modulated signal; $a_{1}(t)$ - instantaneous amplitude; $f_{1}(t)$ - instantaneous frequency; $\varphi(t)$ - instantaneous phase; $\sigma_{i}$ - singular values; $D_{p}$ - pitch diameter; $D_{b}$-diameter of the rolling element; $F_{s}$ - shaft frequency; $\alpha$ - contact angle; $N_{b}$ - number of rolling elements; $F_{B P I}$-inner race ball passes frequency; $F_{B P O}$ - outer race ball passes frequency; $F_{B}$ - Ball Spins Frequency; $F_{C}$ - Cage Frequency.

\section{Introduction}

The health status of rotating machine components can be evaluated by measured information, such as vibrations, without dismantling the machine. Now it is of increasing importance for timely and effective health diagnosis to reduce costly machine downtime and maintain high productivity [1].

There are many techniques of fault characteristic extraction available for the detection of rotating machinery faults such as time-domain statistical analysis, Fourier transform, short-time Fourier transform (STFT), Wigner-Viller distribution (WVD), Wavelet transform (WT) etc [2, 3], but each of these methods has its limitations[4]. For example, the Wigner Ville distribution would cause cross-term interference when dealing with the multi component signals; the analysis window of STFT is fixed; the WT has been well applied in fault diagnosis $[5,6]$ but different mother wavelets should be predefined for each component [7-11].

Vibration based time-frequency analysis to the mechanical vibration signals has become a most successful and effective technique in recent years. Empirical mode decomposition (EMD) has been recently developed in fault diagnosis of rotating machinery. EMD is based on the local characteristic time scales of a signal and could decompose the complicated signal into a set of complete and almost orthogonal intrinsic mode functions (IMFs). EMD is a selfadaptive signal processing method that can be applied to non-linear and non-stationary process perfectly. However, one of the major drawbacks of EMD is the mode mixing problem [12]. In addition, sometimes the unexplainable negative instantaneous frequency would appear when compu- ting instantaneous frequency by performing Hilbert transform to the decomposition results of EMD and meanwhile the end effects would be more serious [13, 14]. A novel selfadaptive time-frequency analysis method named local mean decomposition (LMD), recently, was presented by Jonathan S. Smith [15] and used as a demodulation analysis method, which is particularly suitable for the processing of multicomponent amplitude- modulated and frequency-modulated (AM-FM) signals. By using LMD, any complicated signal can be decomposed into a number of product functions (PFs), each of which is the product of an envelope signal (obtained directly by the decomposition) from which instantaneous amplitude of the PF can be obtained and a purely frequency modulated signal from which a well-defined instantaneous frequency could be calculated. In essence, each $\mathrm{PF}$ is exactly a mono-component AM-FM signal [16].

The procedure of LMD could be, in fact, regarded as the process of demodulation [22-28]. Modulation information can be extracted by performing spectrum analysis to the instantaneous amplitude (envelope signal, obtained directly by the decomposition) of each $\mathrm{PF}$ component rather than by performing Hilbert transform to the PF components. Hence, when LMD and EMD are applied to the demodulation analysis respectively, compared with EMD [17-18], the prominent advantage of LMD is to avoid the Hilbert transform. In addition, the LMD iteration process which uses smoothed local means and local magnitudes avoids the cubic spline approach used in EMD [19], which maybe bring the envelope errors and influence on the precision of the instantaneous frequency and amplitude. Moreover, compared with EMD the end effect is not obvious in LMD approach because of faster algorithm speed and less iterative times [20, 21]. Different researchers have applied LMD combining with other signal processing techniques to bearing fault diagnosis and obtained superior diagnosis results compared with the use of LMD alone [22].

This paper proposes a hybrid approach for the fault detection of rolling element bearing which combine Singular value decomposition (SVD) with LMD algorithm and the results show the excellent performance of the proposed technique in revealing the rolling element bearing fault.

Singular value decomposition (SVD) is a non-parametric technique which has been widely used in feature extraction [23], in voice, image, mobile communication, electric power [24, 25], biomedicine and earthquake monitoring since it was developed to extract the useful element from noisy signal by Tufts. The one-dimensional signal can be transformed into many kinds of matrices, such as Toeplitz matrix, cycle matrix and Hankel matrix. Zhao et al. pointed out that the signal processing effect of Hankel matrix-based 
SVD was very similar to that of wavelet transform. The similarity of Hankel matrix- based SVD and wavelet transform in signal processing could be applied to noise reduction, singularity detection, feature extraction and fault diagnosis [26].

The layout of the paper is as follows: A novel numerical scheme for rolling element bearing fault diagnosis based on hybrid SVD-LMD and conventional signal processing methods is proposed in Section 2. An experimental study is applied for rolling bearing fault diagnosis In Section 3. The results demonstrates that the proposed approach is feasible in Section 4. Finally, the conclusions are presented in Section 5.

\section{The proposed numerical approach for rolling bearing fault diagnosis}

This paper combines SVD and LMD algorithm, the procedure of the numerical approach is briefly described as follows:

Step 1: Implementing the SVD denoising to filter the original signal and separate the signal of interest from the noise.

Step 2: Use LMD method to decompose the purified signal into PFs, meantime instantaneous amplitude and instantaneous frequency of each PF component can be calculated.

Step 3: Denoise the PFs by SVD and sum to LMD again, repeate the same process until we can obtain the precision requisite of the time frequency analysis.

Step 4: Applying spectrum analysis to the instantaneous amplitude of PF component including dominant feature information and extracte the fault characteristic frequency of the roller bearing.

The next Section introduces the basic principle of Local mean decomposition and Singular value dcomposition (SVD).

\subsection{Basic principle of Local mean decomposition}

The steps of LMD of any signal $x(t)$ are as follows [27]:

1. From the original signal $x(t)$, determine all local mean value mi of each two successive extrema $n_{i}$ and $n_{i+1}$ :

$$
m_{i}=\frac{n_{i}+n_{i+1}}{2} .
$$

2. A corresponding envelope estimate $a_{i}$ is given by:

$$
a_{i}=\frac{n_{i}-n_{i+1}}{2} \text {. }
$$

3. Interplate straight lines of local mean and envelope estimate values between successive extrema $m_{i}$ and $a_{i}$, local mean function $m_{11}(t)$ and $a_{11}(t)$ can be formed by using moving averaging to smooth them. original $x(t)$ :

4. Subtract the smoothed mean signal from the

$$
h_{11}(t)=x(t)-m_{11}(t) \text {. }
$$

5 . Get the frequency modulated signal $s_{11}(t)$, by dividing $h_{11}(t)$ by $a_{11}(t)$ :

$$
s_{11}(t)=\frac{h_{11}(t)}{a_{11}(t)} .
$$

6. Check whether $s_{11}(t)$ is a normalized frequencymodulated signal. If $s_{11}(t)$ is a normalized frequency-modulated signal, the envelope function $a_{12}(t)$ is close to 1 . If not, go back to the first step to repeat the same procedure until a purely frequency modulated signal $s_{1 n}(t)$ that meets $-1 \leq s_{1 n}(t) \leq 1$.

So

$$
\left\{\begin{array}{c}
h_{11}(t)=x(t)-m_{11}(t) ; \\
h_{12}(t)=s_{11}(t)-m_{12}(t) ; \\
\cdot \\
\cdot \\
\cdot \\
h_{1 n}(t)=s_{1(n-1)}(t)-m_{1 n}(t),
\end{array}\right.
$$

where

$$
\left\{\begin{aligned}
s_{11}(t) & =\frac{h_{11}(t)}{a_{11}(t)} \\
s_{12}(t) & =\frac{h_{12}(t)}{a_{12}(t)} \\
& \cdot \\
& \cdot \\
& \cdot \\
s_{1 n}(t) & =\frac{h_{1 n}(t)}{a_{1 n}(t)}
\end{aligned}\right.
$$

Where the objective is:

$$
\lim _{\substack{1 n \\ a_{n \rightarrow \infty}}}(t)=1
$$

7. Envelope function $a_{1}(t)$ can be derived by multiplying all $a_{1 k}(t)$ :

$$
a_{1}(t)=a_{11}(t) a_{12}(t) \ldots \ldots a_{1 n}(t)=\prod_{q=1}^{n} a_{1 q}(t) .
$$

8. Using the envelope function $a_{1}(t)$ and the final frequency modulated signal $s_{1 n}(t)$ to get the first product function $P F_{1}$ of the original signal by their multiplication:

$$
P F_{1}(t)=a_{1}(t) s_{1 n}(t) \text {. }
$$

9. The instantaneous amplitude of $P F_{1}$ is $a_{1}(t)$. Instantaneous phase and instantaneous frequency can be determined by (Eq. 10) and (Eq. 11) respectively.

$$
\varphi_{1}(t)=\arccos \left(s_{11}(t)\right) ;
$$




$$
f_{1}(t)=\frac{1}{2 \pi} \frac{d \varphi_{1}(t)}{d t}
$$

10. $P F_{1}$ is subtracted from original data $x(t)$ resulting in a new function $u_{1}(t)$, which becomes the new data and the whole process is repeated $k$ times until $u_{k}(t)$ is a constant or contains no more oscillations.

$$
\left\{\begin{array}{c}
u_{1}(t)=x(t)-P F_{1}(t) \\
u_{2}(t)=u_{1}(t)-P F_{2}(t) \\
\cdot \\
\cdot \\
u_{k}(t)=u_{k-1}(t)-P F_{k}(t) .
\end{array}\right.
$$

Then we let $u_{1}(t)$ as a new original signal and repeat step 1 to step 4 till all $u_{k}(t)$ meet the monotone function condition. Therefore, the original signal can be expressed as the sum of PF components and a monotonic component [31].

$$
x(t)=\sum_{p=1}^{k} P F_{P}(t)+u_{k}(t) .
$$

2.2. Basic principle of Singular Value Decomposition (SVD)

In this paper, SVD is used for denoising vibration signals, by which, the matrix produced with noisy signal is decomposed into a number of singular values and subspaces of signal and noise [28]. The detailed procedures of SVD denoising, can be summarized as follows.

Suppose A is a matrix $m \times n$ whose entries come from the field $K$, which is either the field of real numbers or the field of complex numbers. Then it exists a factorization of the form:

$$
A=U \sum V^{T}
$$

where $U$ is an $\mathrm{m} \times \mathrm{m}$ unitary matrix over $K, \Sigma$ is a $m \times n$ diagonal matrix with non-negative real numbers on the diagonal.

$$
\sum=\left[\operatorname{diag}\left(\sigma_{1}, \sigma_{2}, \ldots . \sigma_{q}\right), 0\right] .
$$

The diagonal entries $\sigma_{i}$ of $\sum$ are known as the singular values $(S V s)$ of $A$. The $n \times n$ unitary matrix $V^{T}$ denotes the conjugate transpose of the $n \times n$ unitary matrix $V$. Such a factorization is called a singular value decomposition of $A$, for a discrete signal $X=[x(1), x(2), \ldots, x(N)]$, commonly, we can construct a Hankel matrix as:

$$
A=\left[\begin{array}{cccc}
x(1) & x(2) & \ldots & x(n) \\
x(2) & x(3) & \ldots & x(n+1) \\
\cdot & \cdot & \cdot & \cdot \\
x(m) & x(m+1) & \ldots & x(N)
\end{array}\right]
$$

And the component matrix Ai when Hankel matrix is used can be formed as:

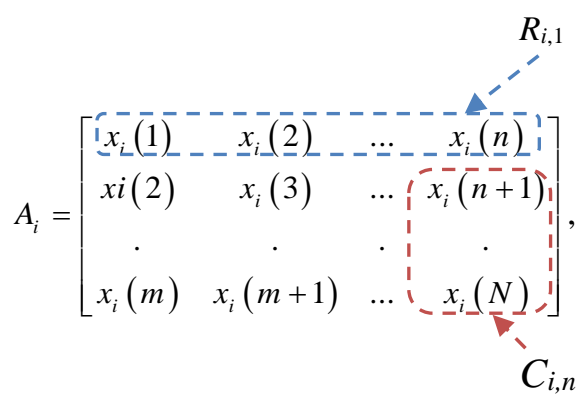

where $1<m<N, \quad n=N-m+1$ and $A \in \mathfrak{R}^{m X n}$. A can be converted as:

$$
A=\sigma_{1} u_{1} v_{1}^{T}+\sigma_{2} u_{2} v_{2}^{T}+\ldots .+\sigma_{q} u_{q} v_{q}^{T}
$$

where $\{u i\}$ and $\{v i\}$, are column vectors of $U$ and $V$, then

$$
A_{i}=\sigma_{i} u_{i} v_{i}^{T}
$$

So:

$$
A=\sum_{i=1}^{q} A_{i}
$$
tor form:

A component signal $P_{i}$ can be expressed as the vec-

$$
P_{i}=\left[R_{i, 1}, C_{i, n}^{T}\right] ; R_{i, 1} \in \mathfrak{R}^{1 X n}, C_{i, n} \in \mathfrak{R}^{(m-1) X 1} .
$$

The original signal can be expressed as:

$$
X=\sum_{i=1}^{q} P_{i}
$$

SVD plays an essential task in signal processing because it can divide a signal space into a preferred space and a useless space, therefore, noisy elements could be subtracted from the received signals. Comparing with the characteristic of noise whose singular values are all close to zero, the singular values of vibration signals collected from a faulty bearing are generally much bigger than zero. So $A$ is the superposition of the vibration signals space and noise space and can be divided into two subspaces as follows:

$$
A=\left[\begin{array}{ll}
U_{s} & U_{N}
\end{array}\right]\left[\begin{array}{cc}
\Sigma_{s} & 0 \\
0 & \Sigma_{N}
\end{array}\right]\left[\begin{array}{ll}
V_{s} & V_{N}
\end{array}\right]^{T} .
$$

So

$$
A=U_{s} \sum_{s} V_{s}^{T}+U_{N} \Sigma_{N} V_{N}^{T}=A_{s}+A_{N},
$$

where

$$
\sum_{s}=\operatorname{diag}\left(\sigma_{1}, \sigma_{2}, \ldots, \sigma_{x}\right)
$$

and

$$
\Sigma_{N}=\operatorname{diag}\left(\sigma_{x+1}, \sigma_{x+2}, \ldots, \sigma_{q}\right),
$$
$\left.\left.\left.\left.\left.\sigma_{1}\right\rangle \sigma_{2}\right\rangle \ldots\right\rangle \sigma_{x}\right\rangle \sigma_{x+1}\right\rangle \ldots \ldots \sigma_{q}$ are the singular values in the signal subspace and the noise subspace. 


$$
\begin{aligned}
& A_{s}=U_{s} \sum_{s} V_{s}^{T} ; \\
& A_{N}=U_{N} \sum_{N} V_{N}^{T} .
\end{aligned}
$$

$A_{s}$ and $A_{N}$ are the signal subspace and the noise subspace, respectively. By subtracting $A_{N}$ from $A$, noisy elements could be suppressed from the received signals, and we can achieve the desired signal.

\section{Experiment data analysis}

To verify the effectiveness of the proposed approach. In this paper all the roller bearing vibration data analyzed are from the website of Case Western Reserve University Bearing Data Center [29], motor power of the test is 14.7 KW, and 6205-2RS SKF bearing was selected, motor speed of test is $1680 \mathrm{tr} / \mathrm{min}$, the sampling frequency is $12000 \mathrm{HZ}$, the test had simulated inner and outer rings fault of the bearing. The fault characteristic frequencies of the roller bearing can be obtained as follows [30, 31]:

Cage frequency $\left(F_{C}\right)$ :

$$
F_{C}=\frac{F_{s}}{2}\left(1-\frac{D_{b}}{D_{p}} \cos \alpha\right),
$$

where $D_{p}$ is the pitch diameter; $D_{b}$ is the diameter of the rolling element; $F_{s}$ is the shaft frequency; $\alpha$ is the contact angle; $N_{b}$ - the number of rolling elements.

The inner race ball passes frequency $\left(F_{B P I}\right)$ :

$$
F_{B P I}=\frac{F_{s}}{2}\left(1+\frac{D_{b}}{D_{p}} \cos \alpha\right) N_{b}
$$

and the outer race ball passes frequency $\left(F_{B P O}\right)$ :

$$
F_{B P O}=\frac{F_{s}}{2}\left(1-\frac{D_{b}}{D_{p}} \cos \alpha\right) N_{b} .
$$

Ball spins frequency $\left(F_{B}\right)$ :

$$
F_{B}=\frac{F_{s}}{2} \frac{D_{p}}{D_{b}}\left(1-\left(\frac{D_{b}}{D_{p}} \cos \alpha\right)^{2}\right) N_{b}
$$

Bearing specification

\begin{tabular}{|c|c|c|c|}
\hline$D_{p}, \mathrm{~mm}$ & $D_{b}, \mathrm{~mm}$ & $N_{b}$, ball & $\alpha$, degree \\
\hline 39.04 & 7.94 & 9 & 0 \\
\hline
\end{tabular}

The running speed of the motor was $28 \mathrm{~Hz}$, based on the bearing parameters given in Table, the characteristic frequency of the roller bearing with outrace fault is calculated as $F_{B P O}=107.57 \mathrm{~Hz}$, the characteristic frequency of the roller bearing with inner-race fault is calculated as $F_{B P I}=162.45 \mathrm{~Hz}$.

\section{Results and discussion}

Fig. 1 show the raw vibration signals extracted from a healthy bearing. In industrial environment, informative accelerations are always affected, buried and masked by noises. Subsequently, the application of LMD for roller bearing vibration signals processing produces some PFs also strongly affected by noises. In other words, the number of the decomposed PFs is dependent on the noise quantity.

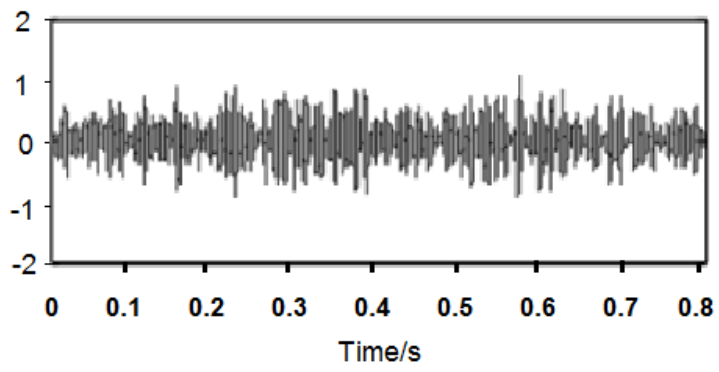

Fig. 1 The raw vibration signal collected from a healthy bearing

By applying the proposed approach, we can see the number of PFs derived from the original signals, shown as Fig. 2. After that, the spectrum analysis using FFT transform is applied to the instantaneous amplitude of PF1
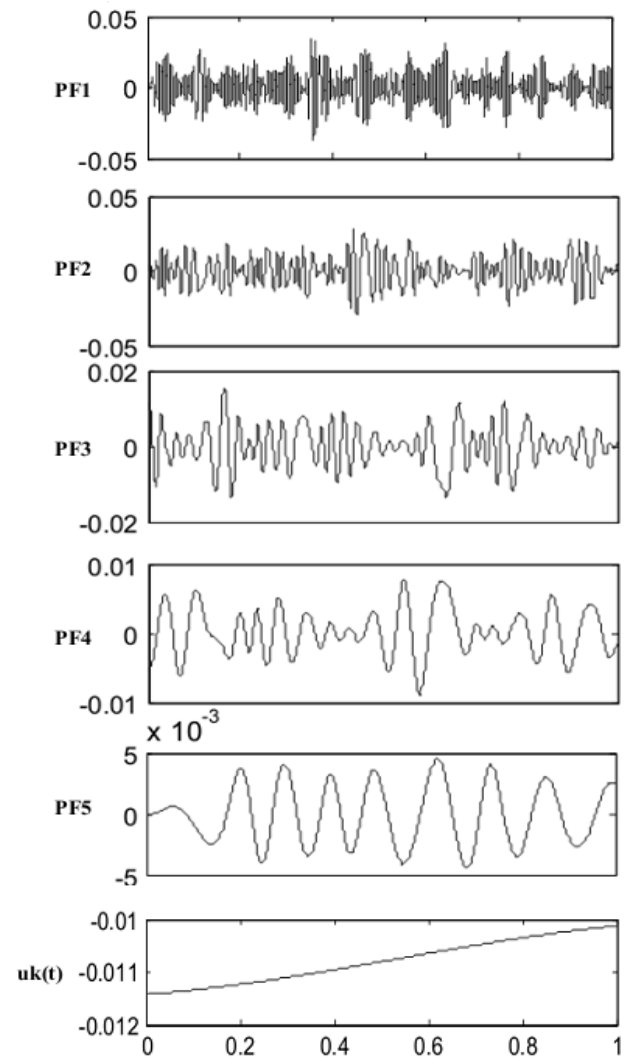

Fig. 2 PFs of healthy bearing using the proposed method

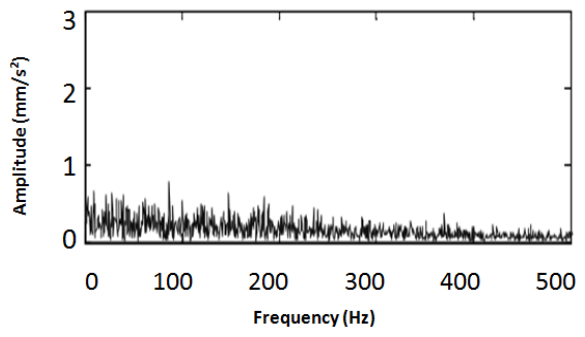

Fig. 3 The FFT spectrum of the instantaneous amplitude of PF1 
component to extract the characteristic frequency of the roller bearing. When normal bearing data are analyzed using the proposed method, from the results obtained in Fig. 3, no fault characteristic frequency and its harmonics can be identified.

Fig. 4 shows the raw vibration signal collected from bearing with an outer race fault. The impulses related to the features of the faulty bearings were almost completely masked by noise, it can be seen that due to the defect present in the rolling bearing, the vibration signal presents the periodicity impacts features, but there exist very serious ambient noises.

Consequently, we combine LMD with other techniques to bearing fault diagnosis.

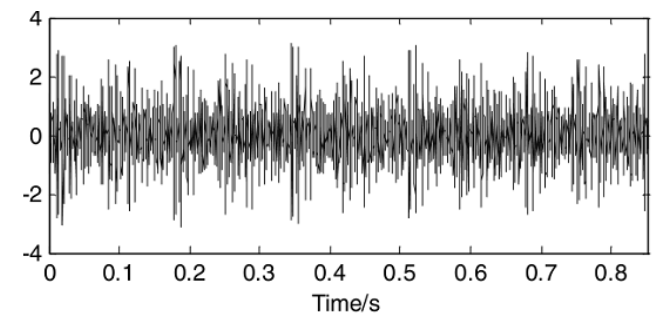

Fig. 4 The raw vibration signal collected from bearing with an outer race fault

With LMD method, the vibration acceleration signal is decomposed to $5 \mathrm{PF}$ components and one residue shown in Fig. 5. Obviously, PF1 is still very complicated, even similar to the original vibration signal, it can be observed in Fig. 6 that the fault feature is drowned by the background signals relevant to the rotary speed of rotor and other noise, the FFT spectra cannot capture and illustrate any fault characteristics.

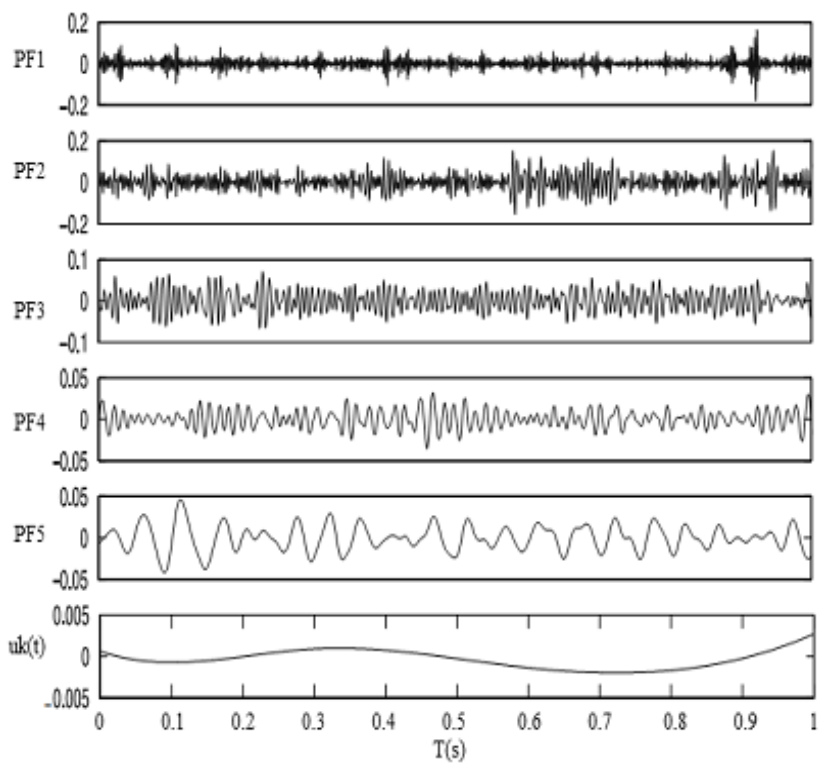

Fig. 5 PFs of tested bearing with outrace fault obtained by LMD

Applying the proposed method which combines SVD denoising and LMD algorithm. The ambient noises are effectively suppressed, meanwhile, the periodicity impacts features are well reserved, PFs components are obtained shown in Fig. 7. Meantime instantaneous amplitude and instantaneous frequency of each PF component can be calcu- lated then fault feature can be extracted accurately by applying spectrum analysis to the instantaneous amplitude of PF component including dominant feature information.

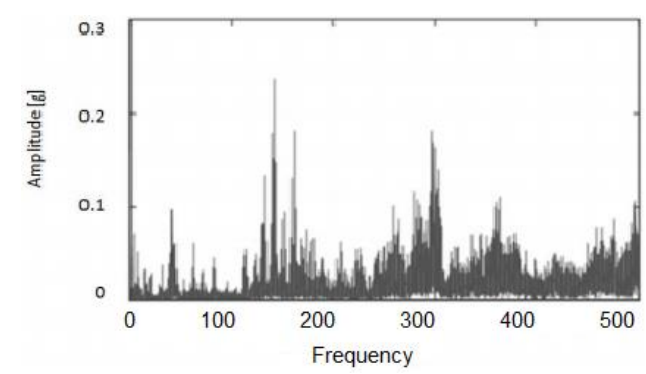

Fig. 6 The FFT spectrum of the instantaneous amplitude of PF1

From Fig. 7, it can be seen clearly PF1 is modulated, so the fault feature of vibration signals would be extracted effectively by applying FFT transform to the instantaneous amplitude of the first PF component.

The calculated frequency of the roller bearing with outrace fault $(107.57 \mathrm{~Hz})$ and its harmonic frequencies could be found in the amplitude spectrum shown in (Fig. 8), it is clear that the proposed method can achieve better results compared with the use of LMD alone.

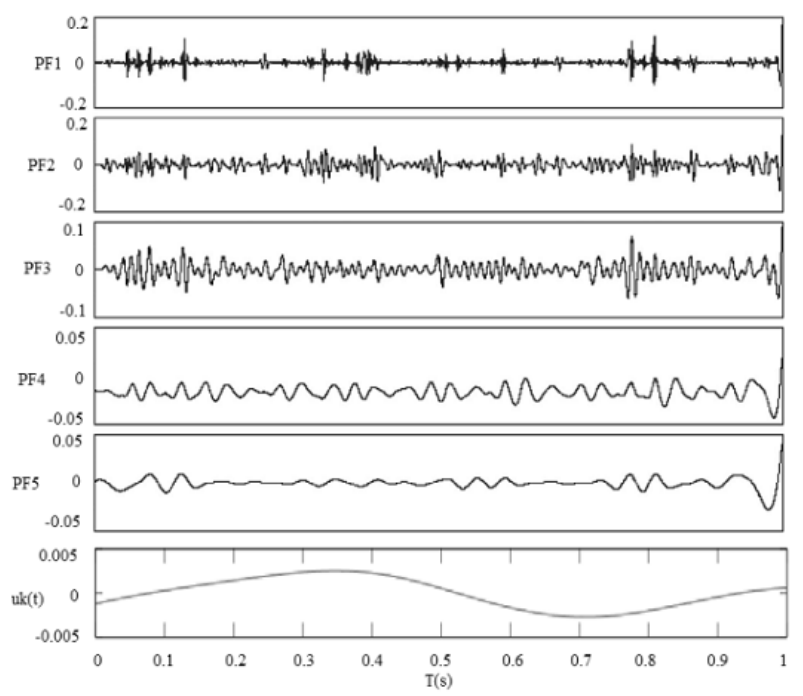

Fig. 7 PFs of tested bearing with outrace fault using hybrid SVD and LMD

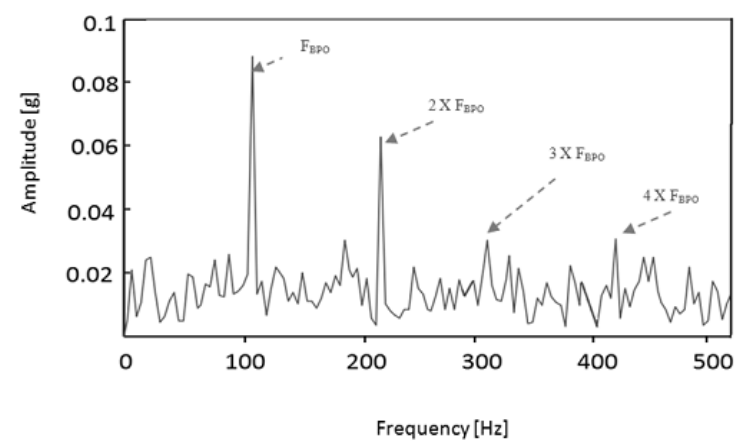

Fig. 8 The FFT spectrum of the instantaneous amplitude of PF1

Fig. 9 shows the raw vibration signals of defective bearing with an inner race fault. The theoretic characteristic 


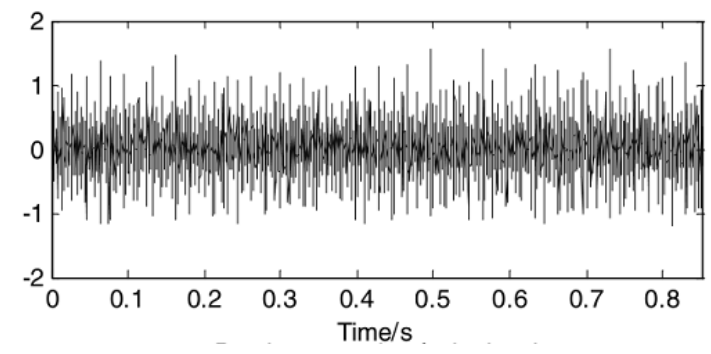

Fig. 9 The raw vibration signal collected from bearing with inner race fault

frequency of the roller bearing with inner-race fault is calculated as $F_{B P I}=162.45 \mathrm{~Hz}$.

By applying hybrid SVD-LMD the PFs components are obtained, shown as Fig. 10. After that, the spectrum analysis using FFT transform is applied to the instantaneous amplitude of PF1 component to extracte the demodulated frequency. The theoretic characteristic frequency of the roller bearing with inner-race fault $(162.45 \mathrm{~Hz})$ and the shaft rotational frequency and its third harmonic have been clearly detected in the amplitude spectra shown in Fig. 11, which indicates the amplitude modulation of the carrier frequency by the defect frequencies. That is exactly the characteristics of vibration signal when the roller bearing has inner-race fault.

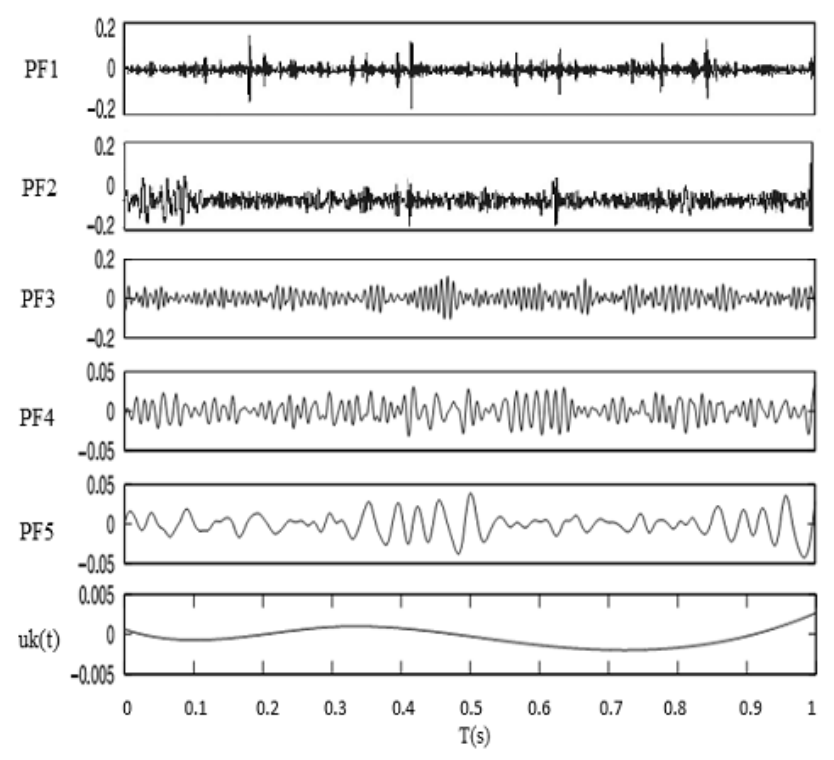

Fig. 10 PFs of tested bearing with inner race defect using hybrid SVD and LMD

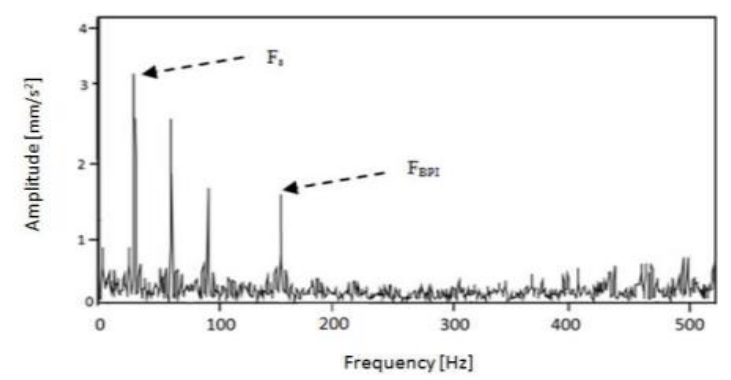

Fig. 11 The FFT spectrum of the instantaneous amplitude of PF1

\section{Conclusions}

When bearing is at fault, the vibration signal is usually many components of the complex modulation signal. In this paper, we have proposed a numerical approach for rolling element bearing fault diagnosis, which combine SVD denoising with LMD algorithm. LMD is very suitable for the analysis and feature extraction for non-stationary modulation signals. SVD is taken as the as prefilter to denoise and enhance the impulsive features, then the fault characteristic frequency of the roller bearing can be extracted by applying spectrum analysis to the instantaneous amplitude of PF component containing dominant fault information. Finally the results demonstrate that the proposed approach is successful for rolling bearing fault detection and diagnosis.

\section{Acknowledgments}

The authors thank Case Western Reserve University for providing the Bearing Fault Data Files freely over the web.

\section{References}

1. Dong, Y.B.; Liao, M.F.; Zhang, X.L. 2011. Faults diagnosis of rolling element bearings based on modified morphological method, Mech. Syst. Signal Process 25(4): 1276-1286.

http://dx.doi.org/10.1016/j.ymssp.2010.10.008.

2. Lei, Y.G.; Lin, J.; He, Z.J. 2011. Application of an improved kurtogram method for fault diagnosis of rolling element bearings, Mech. Syst. Signal Process 25(1): 1738-1749. http://dx.doi.org/10.1016/j.ymssp.2010.12.011.

3. Zhu Fulei; Peng Zhike; Feng Zhipeng 2009. Mechanical fault diagnosis of modern signal processing methods, Beijing Science Press 41(4): 1-130.

4. Fan, X.; Liang, M.; Yeap, T.H.; Kind, B. 2007. A joint wavelet lifting and independent component analysis approach to fault detection of rolling element bearing, Smart Mater. Struct. 16(5): 1973-1987. http://dx.doi.org/10.1088/0964-1726/16/5/056.

5. He, W.; Jiang, Z.N.; Qin, Q. 2010. A joint adaptive wavelet filter and morphological signal processing method for weak mechanical impulse extraction, J. Mech. Sci. Technol. 24(8): 1709-1716. http://dx.doi.org/10.1007/s12206-010-0511-4.

6. Li, C.J.; Ma, J. 1997. Wavelet decomposition of vibrations for detection of bearing-localized defects, NDT\&E Int. 30(3): 143-149. http://dx.doi.org/10.1016/S0963-8695(96)00052-7.

7. McFadden, P.D.; Wang, W.J. 1992. Analysis of gear vibration signatures by the weighted Wigner-Ville distribution, Inter. Conference on Vibrations in Rotating Machinery, Institution of Mechanical Engineers, Bath, 387-393.

8. Miao, Q.; Huang, H.Z.; Fan, X.F. 2007. Singularity detection in machinery health monitoring using Lipschitz exponent function, J. Mech. Sci. Technol. 21(5): 737-744. http://dx.doi.org/10.1007/BF02916351.

9. Mori, K.; Kasashima, N.; Yoshioka, T.; Ueno, Y. 1996. Prediction of spalling on a ball bearing by applying the discrete wavelet transform to vibration signals, 
Wear 195(1): 162-168

http://dx.doi.org/10.1016/0043-1648(95)06817-1.

10. Mallat, S. 1989. A theory for multiresolution signal decomposition. The wavelet representation, IEEE T Pattern Anal 11(7): 674-693. http://dx.doi.org/10.1109/34.192463.

11. Fan, X.; Liang, M.; Yeap, T.H.; Kind, B. 2007. A joint wavelet lifting and independent component analysis approach to fault detection of rolling element bearings, Smart Mater. Struct. 16(5): 1973-1987. http://dx.doi.org/10.1088/0964-1726/16/5/056.

12. Zhang, X.Y.; Zhou, J.Z. 2013. Multi-fault diagnosis for rolling element bearings based on ensemble empirical mode decomposition and optimized support vector machines, Mech. Syst. Signal Process 41(1): 127-140. http://dx.doi.org/10.1016/j.ymssp.2013.07.006.

13. Yang, Y.; Yu, D.J.; Cheng, J.S. 2007 .A fault diagnosis approach for roller bearing based on IMF envelope spectrum and SVM, Measurement 40(9): 943-950. http://dx.doi.org/10.1016/j.measurement.2006.10.010.

14. Tandon, N.; Choudhury, A. 1999. A review of vibration and acoustic measurement methods for the detection of defects in rolling element bearings, Tribol. Int. 24(3): 469-480. http://dx.doi.org/10.1016/S0301-679X(99)00077-8.

15. Smith, Jonathan S. 2005. The local mean decomposition and its application to EEG perception data, Journal of the Royal Society, Interface 2(5): 443-454. http://dx.doi.org/10.1098/rsif.2005.0058.

16. Wang, Y.; He, Z.; Zi, Y. 2010. A comparative study on the local mean decomposition and empirical mode decomposition and their applications to rotating machinery health diagnosis, J. Vib. Acoust. 132(2): 021-010.

17. Ricci, R.; Pennacchi, P. 2011. Diagnostics of gear faults based on EMD and automatic selection of intrinsic mode functions, Mechanical Systems and Signal Processing 25(3): 821-838. http://dx.doi.org/10.1016/j.ymssp.2010.10.002.

18. Lei, Y.; Lin, J.; He, Z.; Zuo, M.J. 2013. A review on empirical mode decomposition in fault diagnosis of rotating machinery, Mech. Syst. Signal Process 35(1-2): $108-126$. http://dx.doi.org/10.1016/j.ymssp.2012.09.015.

19. Cheng, J.S.; Yang, Y.; Yang, Y. 2012. A rotating machinery fault diagnosis method based on local mean decomposition, Digital Signal Processing 22(2): 356-366. http://dx.doi.org/10.1016/j.dsp.2011.09.008.

20. Chen, B.J.; He, Z.J.; Chen, X.F. 2011. A demodulating approach based on local mean decomposition and its applications in mechanical fault diagnosis, Measurement Science and Technology 22(5): 055-704.

21. Wang, Y.X.; He, Z.J.; Xiang, J.W.; Zi, Y.Y. 2012. Application of local mean decomposition to the surveillance and diagnostics of low-speed helical gearbox, Mechanism and Machine Theory 47(4): 62-73. http://dx.doi.org/10.1016/j.mechmachtheory.2011.08.007.

22. Yang, Y; Cheng, J.S.; Zhang, K. 2012. An ensemble local means decomposition method and its application to local rub-impact fault diagnosis of the rotor systems, Measurement 45(3): 561-570. http://dx.doi.org/10.1016/j.measurement.2011.10.010.

23. Delponte, E.; Isgro, F.; Odone, F.; Verri, A. 2006. SVD-matching using SIFT features, Graph. Models
68(6): 415-431.

http://dx.doi.org/10.1016/j.gmod.2006.07.002.

24. Dong, S.J.; Tang, B.P.; Zhang, Y. 2012. A repeated single-channel mechanical signal blind separation method based on morphological filtering and singular value decomposition, Measurement 45(8): 2052-2063. http://dx.doi.org/10.1016/j.measurement.2012.05.003.

25. Hu, A.J.; Sun, J.J.; Xiang, L. 2012. Analysis of morphological filter's frequency response characteristics in vibration signal processing, Chin. J. Mech. Eng. 48(1): 98-103. http://dx.doi.org/10.3901/JME.2012.01.098.

26. Wang, D.; Peter, W.T.; Guo, W.; Miao, Q. 2011. Support vector data description for fusion of multiple health indicators for enhancing gearbox fault diagnosis and prognosis, Meas. Sci. Technol. 22(2). http://dx.doi.org/10.1088/0957-0233/22/2/025102.

27. Hu Jin Song; Yang Shi; Xi Ren Daqian 2009. Splinebased local mean decomposition method for vibration signal, Journal of Data Acquisition \& Processing 24(1): 82-86 (in Chinese).

28. Zhao, F. 2004. Image matching based on singular value decomposition, Proceedings of the 5th Pacific Rim Conference on Multimedia (PCM), Tokyo, Japan, 119-126.

29. Case Western Reserve University Bearing Data Center. 2011. http://csegroups.case.edu/bearingdatacenter/pages /download-data-file.

30. Zhu Fulei; Peng Zhike; Feng Zhipeng 2009. Mechanical fault diagnosis of modern signal processing methods, Beijing: Science Press, 1-130.

31. Zhang, L.; Xiong, G.; Liu, H.; Zou, H.; Guo, W. 2010. Bearing fault diagnosis using multi-scale entropy and adaptive neuro-fuzzy inference, Expert Syst. Appl. 37(60): 6077-6085.

http://dx.doi.org/10.1016/j.eswa.2010.02.118.

\section{T. Bensana, S. Mekhilef}

\section{NUMERICAL AND EXPERIMENTAL ANALYSIS OF VIBRATORY SIGNALS FOR ROLLING BEARING FAULT DIAGNOSIS}

$\mathrm{S} \mathrm{u} \mathrm{m} \mathrm{m} \mathrm{a} \mathrm{r} \mathrm{y}$

The detecting technique of the mechanical anomaly has been a hot topic in the scientific and engineering community. Vibration analysis has been frequently applied in the condition monitoring and fault diagnosis of rolling element bearings. Unfortunately, the vibration signals collected from a faulty bearing are generally nonstationary, nonlinear and with strong noise interference, so it is essential to obtain the fault features correctly. In this paper, a novel numerical analysis method that combines the singular value decomposition (SVD) and local mean decomposition (LMD) is proposed. SVD is a non-parametric technique which has been widely used to eliminate the noise and enhance the impulsive features, then LMD decompose the purified signal into a series of product functions (PFs), each of which is the product of an envelope signal and a purely frequency modulated FM signal. The envelope of a PF is the instantaneous amplitude (IA) and the derivative of the unwrapped phase of a purely flat frequency demodulated (FM) signal is the IF. After that the fault characteristic frequency 
of the roller bearing can be extracted by performing spectrum analysis to the instantaneous amplitude of PF component containing dominant fault information. Finally, the proposed method is applied to experimental data and the results show the effectiveness of the proposed technique in fault detection and diagnosis of rolling element bearing.
Keywords: fault diagnosis, vibration analysis, rolling element bearing.

Received April 16, 2015

Accepted May 11, 2016 